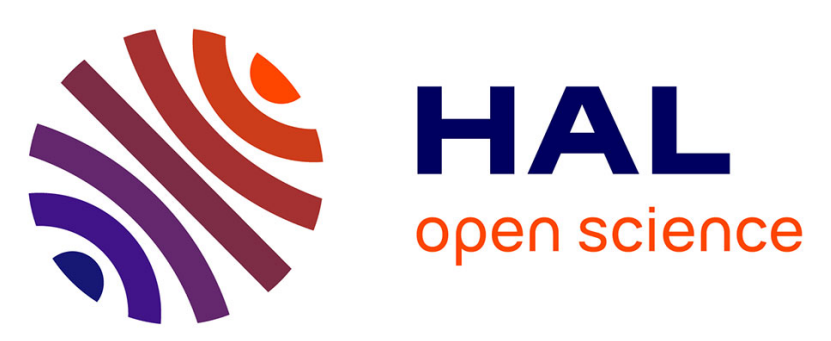

\title{
Adding a protective K-wire during opening high tibial osteotomy increases lateral hinge resistance to fracture
}

Edouard Dessyn, Akash Sharma, Mathias Donnez, Patrick Chabrand, Matthieu Ehlinger, Jean-Noël Argenson, Sebastien Parratte, Matthieu Ollivier

\section{- To cite this version:}

Edouard Dessyn, Akash Sharma, Mathias Donnez, Patrick Chabrand, Matthieu Ehlinger, et al.. Adding a protective K-wire during opening high tibial osteotomy increases lateral hinge resistance to fracture. Knee Surgery, Sports Traumatology, Arthroscopy, 2020, 28 (3), pp.751-758. 10.1007/s00167-019-05404-7 . hal-03176966

\section{HAL Id: hal-03176966 https://hal.science/hal-03176966}

Submitted on 14 Apr 2021

HAL is a multi-disciplinary open access archive for the deposit and dissemination of scientific research documents, whether they are published or not. The documents may come from teaching and research institutions in France or abroad, or from public or private research centers.
L'archive ouverte pluridisciplinaire HAL, est destinée au dépôt et à la diffusion de documents scientifiques de niveau recherche, publiés ou non, émanant des établissements d'enseignement et de recherche français ou étrangers, des laboratoires publics ou privés. 


\title{
Adding a protective K-wire during opening high tibial osteotomy increases lateral hinge resistance to fracture
}

\author{
Edouard Dessyn ${ }^{1,3} \cdot$ Akash Sharma $^{1,3} \cdot$ Mathias Donnez $^{1,3} \cdot$ Patrick Chabrand $^{1,3} \cdot$ Matthieu Ehlinger $^{2}$. \\ Jean-Noël Argenson ${ }^{1,3}$. Sebastien Parratte ${ }^{1,3} \cdot$ Matthieu Ollivier ${ }^{1,3}$
}

\begin{abstract}
Purpose It was hypothesized in this in-vitro study that positioning a K-wire intersecting the cutting plane at the theoretical lateral hinge location would limit the cut depth and help preserve the lateral hinge during the opening of the osteotomy. Objectives were (1) to compare the mechanical resistance of the hinge and the protective effect of leaving the K-wire during the opening procedure (2) to check if the K-wire would limit the depth of the osteotomy.

Methods An ex-vivo mechanical study, testing 5 pairs of fresh-frozen tibias, was designed. CT-scan based Patientspecific cutting guides were obtained to define the cutting plane and the location of the K-wire at the hinge, using standardized 3D planning protocol. In each pair, OWHTO was performed either with or without the K-wire. To evaluate the hinge's resistance to fracture, the specimens were rigidly fixed at the proximal tibia and a direct load was applied on the free tibial diaphysis to open the osteotomy. The maximum load at breakage, maximum permissible displacement and maximal angulation of the osteotomy before hinge failure was measured. To assess the preservation of an unscathed hinge (protected by the K-wire), the distance from the end of the osteotomy cut to the lateral tibial cortical was measured in $\mathrm{mm}$.

Results The maximum load to hinge breakage in the K-wires PsCG knees compared to the control group (48.3 N vs 5.5 $\mathrm{N}, p=0.004)$, the maximum permissible displacement ( $19.8 \mathrm{~mm}$ vs $7.5 \mathrm{~mm}, p=0.005)$ and the maximal angulation of the osteotomy before hinge breakage $\left(9.9^{\circ}\right.$ vs $\left.2.9^{\circ}, p=0.002\right)$ were all statistically superior in the K-wires PsCG knees compared to the control group. A mean distance of $10 \pm 1 \mathrm{~mm}$ between cut-bone (saw-print) and lateral hinge cortical bone was found post-performing the osteotomy and the hinge failing.

Conclusion The maximum load to breakage and the maximum permissible displacement were, respectively, $880 \%$ and $260 \%$ higher during the opening of the OWHTO in using K-wires compared to the non-K-wire control group. This confirms the mechanical advantage of using a K-wire for both stabilization and protecting the Hinge during OWHTO. This comparative cadaveric study shows an improvement of the lateral hinges resistance to failing during the opening of the osteotomy. This can be achieved by the placement of a K-wire intersecting the cutting plane at the theoretical location of the lateral hinge.
\end{abstract}

Keywords High tibial valgus osteotomy $\cdot$ Patient-specific cutting guides $\cdot$ Hinge $\cdot$ Maximum load, cadaveric study

This work was performed at the Institute for Locomotion, AixMarseille University, Marseille, France.

Matthieu Ollivier

matthieu.ollivier@ap-hm.fr

1 Department of Orthopedics and Traumatology, Institute of movement and locomotion, St Marguerite Hospital, 270 Boulevard Sainte Marguerite, BP 29, 13274 Marseille, France

2 Service de Chirurgie Orthopédique et de Traumatologie, CHU Hautepierre, Hôpital de Hautepierre, Hôpitaux Universitaires de Strasbourg, 1 Avenue Molière, 67098 Strasbourg Cedex, France
Department of Orthopedics and Traumatology, Institute for Locomotion, Aix Marseille Univ, APHM, CNRS, ISM, Sainte-Marguerite Hospital, Marseille, France 


\section{Introduction}

Open Wedge High Tibial Osteotomy (OWHTO) remains an established surgical procedure $[1,7,18]$ for young and active patients with medial unicompartmental knee osteoarthritis secondary to varus mal-alignment of the lower limb. The success of an OWHTO depends on primarily following the correct indications, performing an accurate correction and maintaining the angulation and stabilization with a chosen method of an internal fixation, whilst keeping an intact lateral hinge $[8,19,25]$. Amongst these governing facets, preserving the lateral hinge plays a crucial role in improving postoperative outcomes, facilitating bone healing and stabilizing the osteotomy $[3,5,10$, 22]. If damaged, the fixation may not be sufficient and a secondary loss of correction may occur $[3,5,10,22]$. Takeuchi et al. [26] described a classification of hinge fractures in OWHTO. In their study, the authors reported an incidence of $25 \%$ for lateral tibial hinge fractures during OWHTO. Therefore, finding a solution to preserve the hinge unscathed seems critical to prevent a rather problematic consequence for both the patient and surgeon. Some Clinicians have tried to monitor their cut and opening using fluoroscopic images and protective cutting systems $[16,17]$. Others have studied the influence of drilling the hinge through the saw cut to reduce the mechanical stress passing through the lateral tibial hinge [6]. Computer-assisted surgery (CAS) can also offer solutions to the surgeon by controlling the depth of the cut, however this alone does not prevent the hinge to be susceptible to fracture [24].

In a previous study, Munier et al. [20] demonstrated the use of patient-specific cutting guides (PsCG) offers an easy, reliable and reproducible procedure to create and maintain an optimal correction in all 3 planes during OWHTO. It was the aim of this research project to evaluate if PsCG could also offer an easy and reliable method to protect the lateral tibial hinge and increase its resistance to fracture. The hypothesis of this in-vitro study was that positioning a $\mathrm{K}$-wire to intersect the cutting plane at the theoretical lateral hinge location as a result of using a PsCG would limit the cut depth and augment the strength of the lateral hinge during the opening. Using PsCG dedicated to OWHTO [20], a comparative in-vitro study was set up using five matched pairs of cadaveric knees. Objectives were first to study the protective effect on the hinge's resistance to fracture when a $\mathrm{K}$-wire was left in the lateral tibial hinge during the opening procedure and secondly to check if the K-wire would limit the cut depth. To our knowledge, a majority of published series focus on the diagnosis and treatment of OWHTO hinge fracture, and few provided reliable preventative measures to limit them, making this study original.

\section{Materials and methods}

Five matched pairs of knees ( 3 females and 2 males aged from 75 to 97, average age 88.8) were obtained from AixMarseille University Anatomical Department. Anatomical and biomechanical labs scientific and ethic committees validated our study protocol and ensured ethical use of de-identified specimens. Bones were completely dissected leaving only the patellar tendon insertion intact to allow positioning of the cutting guide [20]. All specimens underwent a standardized CT-scan protocol (Discovery 710, GE Medical System, CERIMED, Marseille, France). 3D geometrical models of the tibias were created using Mimics 17.0 software (Materialise ${ }^{\circledR}$, Leuven, Belgium). The 3D models were used to design a patient-specific cutting guide for each tibia [20], defining the cutting plane and the insertion of two $\varnothing 2.2 \mathrm{~mm} \mathrm{~K}$-wires locations. It is accepted that the osteotomy cut should stop approximately $10 \mathrm{~mm}$ from the lateral cortex [20]. The appropriate positioning of the hinge is now known for prevention of an unstable lateral hinge fracture [21]. To maintain and protect the lateral tibial hinge from an inadvertent cut from the sawblade, the first K-wire (Cut K-wire) was parallel and just below the cutting plane, whilst the second (Hinge's K-wire: HK) was placed from distal to proximal from antero-medial to lateral to intersect the cutting plane at the theoretical hinge point (10 $\mathrm{mm}$ from the lateral cortex). The Cut K-wire was used to control the accurate positioning of patient-specific cutting guide, and to maintain the guide rigidly to the bone during the process of drilling the pin holes. Importantly, it also helps in guiding the sawblade during the second part of the cut when the guide is taken off. The PsCG has 4 drill guides: 2 proximal and 2 distal to the cutting plane to improve the PsCG stability using $\varnothing 3.9 \mathrm{~mm}$ pins. The two holes proximal to the cutting plane were also made to predict the position of the tibias on the experimental setup and to increase the rigidity for mechanical testing as described later. For each tibia, the PsCG was placed on the antero-medial tibial cortex. K-wires were inserted, and the four holes were drilled to insert the $\varnothing 3.9 \mathrm{~mm}$ pins. The osteotomy was then performed and the cut was limited by the the buttress in the slot for the saw guide. The thickness of the sawblade used was $1.27 \mathrm{~mm}$. The next step was to remove the PsCG including the pins except for the HK, the cut was then extended until the saw touches the HK (Fig. 1).

The proximal tibial epiphysis was fixed in PVC tubes using two rods inserted through the tubes and through the two proximal drill holes proximal to the cutting plane. The location of the rods was pre-planned on 3D models to have the resin parallel to the cutting plane, to ensure a reproducible position of the tibia inside the PVC tubes. The proximal tibial epiphysis was embedded in PVC tubes with Fascast polyurethane resin (Axson Technologies, Cergy, France). 


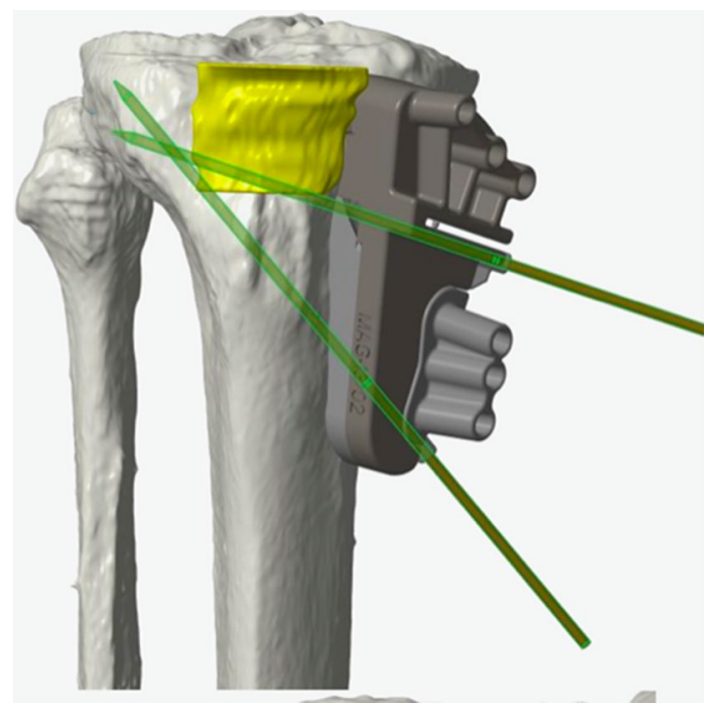

Fig. $13 \mathrm{D}$ schematization of a tibia showing the crossing of the two K-wires: the upper one parallel and just below to the cutting plane, the second one ascendant from antero-medial to lateral that intersect the cutting plane at a theoretical hinge point, identifying the lateral tibial hinge that would be protected from the saw

All the tibias followed the same preparation process with the same parameters (cutting plane location and same Hinge pin location in all tibias). The experimental conditions were strictly identical and reproductible for each pair of tibial specimen being tested. The positioning of the PsCG, the creation of the osteotomy cut, the fixation of the tibia in the experimental setup, the anatomical location of the osteotomy cut and its depth in the epiphyseal tibia was strictly the same. Two factors only were modified in each pair of tibia: the lateralization of the tibia (right vs left) and the maintenance or the removal of the HK during OWHTO.
For mechanical testing, two groups were defined: in each matched pair, the HK was removed from only one tibia randomly chosen, defining the two groups: a "HK group (HK+) and a "No HK" group (HK-). To accurately reproduce surgical opening, the tibial epiphysis was fixed and a load was applied on the tibial diaphysis. When ready, tibias were placed in an Instron loading platform (INSTRON 5566A, Norwood, MA, USA). Load was applied on the diaphysis in a single load-to-failure test. Quasi-static loading with prescribed displacements at $10 \mathrm{~mm} / \mathrm{min}$ was applied up until failure, according to a previously published protocol [26] (Fig. 2). For every tibia, the presence of a unscathed lateral tibial hinge was visually assessed before the testing. Visually, the hinges were classified as broken or not in each group. For each tibia after fixation within the experimental setup, opening angles, vertical displacement in $\mathrm{mm}$ and load applied in $N$ on the diaphysis was recorded. Failure was considered just before a significant drop occurred on the Force/Displacement curve. Force at failure (ML) and corresponding displacement (DML) were identified. Opening angles at failure $\alpha$ were also calculated based on DML value (Eq. 1, Fig. 3):

$\alpha=\alpha 1-\alpha 2=\left(\tan ^{(-1)}((y / x))-\left(\tan ^{(-1)}((\mathrm{DML}-y) / x)\right)\right.$.

In each pair, the influence of the HK was evaluated by comparing DML, ML and $\alpha$ between the $\mathrm{HK}+$ and the HK-. All tests were carried out until the fracture of the lateral hinge. The Load-Displacement curves were available for each tibia (Fig. 4a, b). Based on 3D models used to design the PsCG, distances $x, y, z$ were measured and the opening angles at failure were calculated using this method of reading the graphical coordinates of the ML and the DML. After Mechanical testing including fracture of the lateral hinge, an evaluation of the distal part of the

Fig. 2 Final experimental model in the experimental device with application on the tibial diaphysis of the load and start of the two cameras to film the experiment

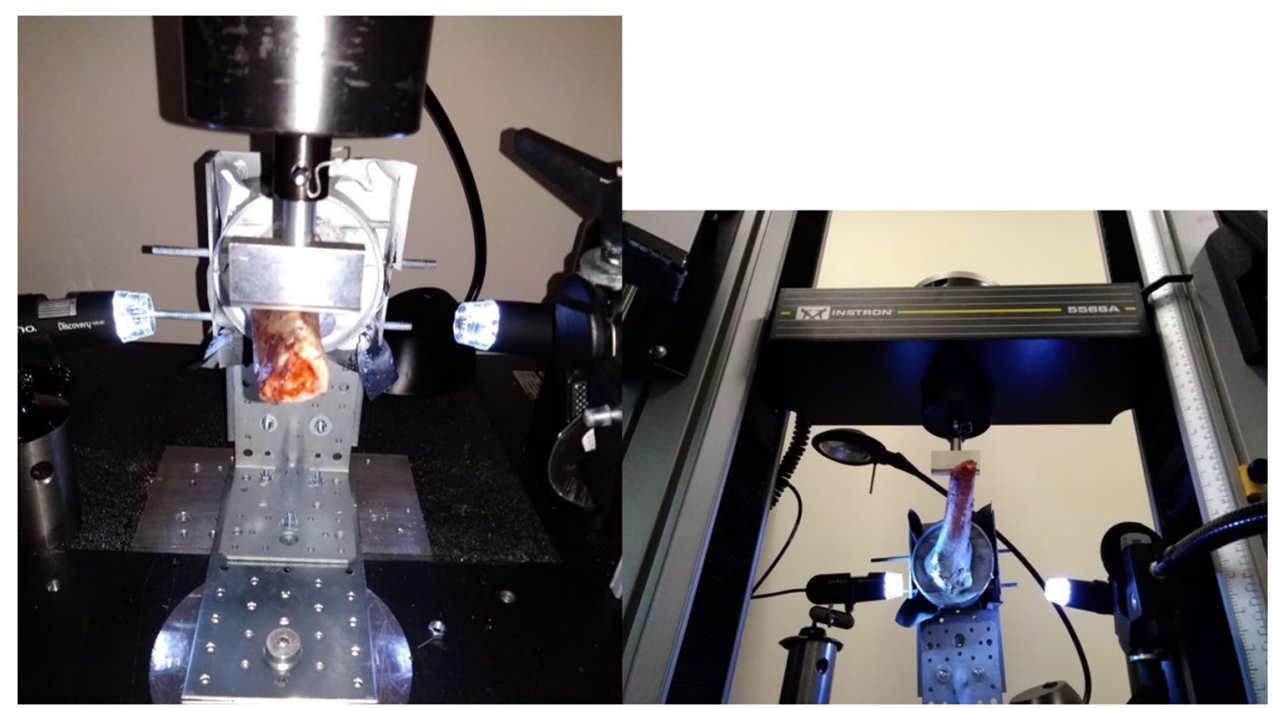


Fig. 3 Schematic representation of $\alpha, x, y$, and $z$
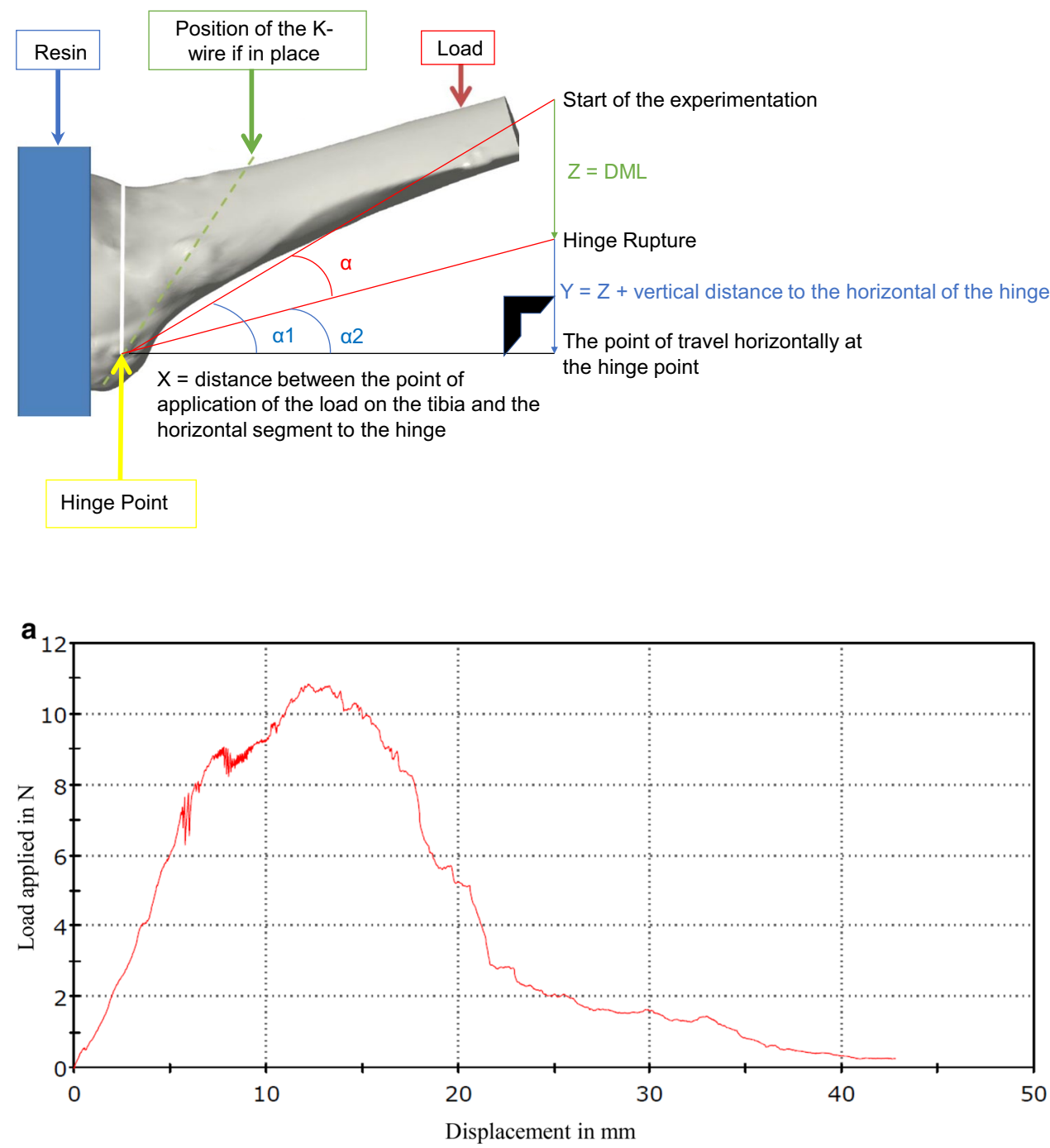

b

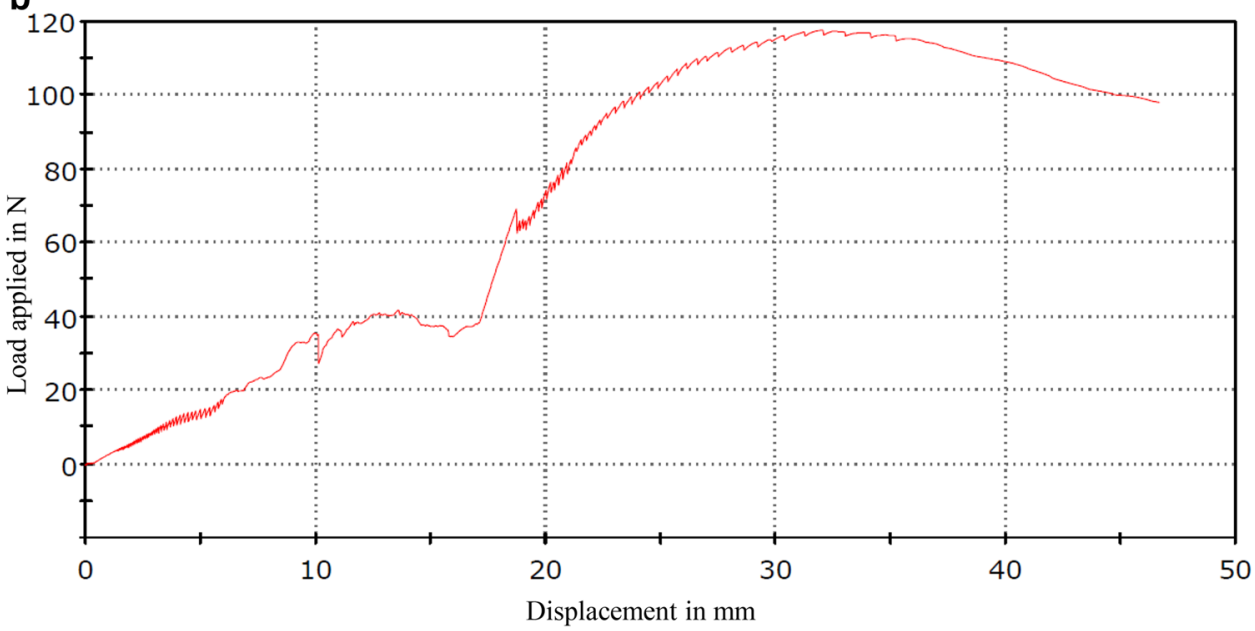

Fig. 4 a Graphic results of ML and DML applied to an HK-knee. b Graphic results of ML and DML applied to a $\mathrm{HK}+\mathrm{knee}$ osteotomy was made by 2 of the authors (ED and MD). The purpose of this evaluation was to measure, using a caliper, the distance in mm between cut (saw-print) and the lateral aspect of hinge's cortical bone (Fig. 5). The intra and extra-observer correlation was evaluated using the Spearman test. One of the authors (trained engineer: MD) analysed the results on our load-displacement curves. The precision reported was fixed to one 
Fig. 5 Measurement of the distance between cut-bone and hinge lateral cortical

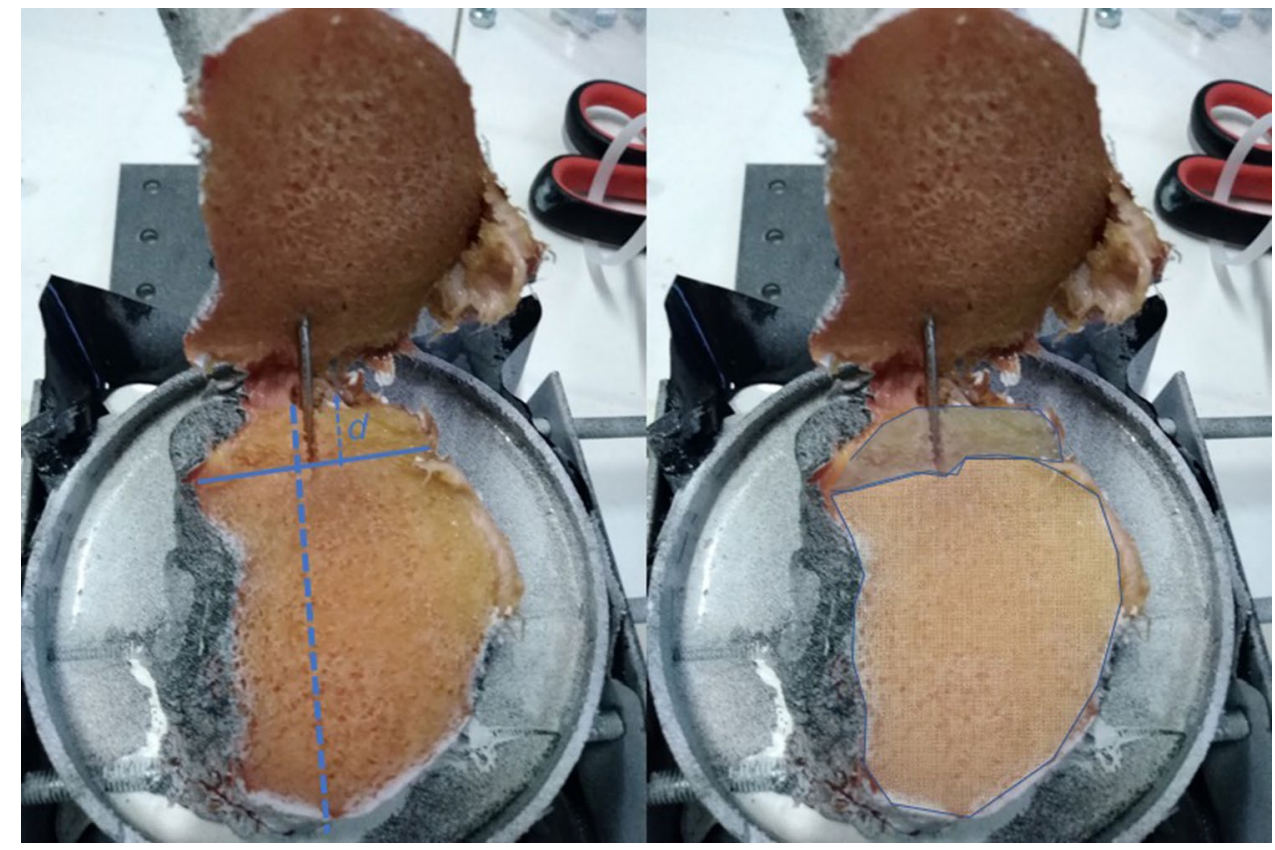

decimal for the DML $(0.1 \mathrm{~mm})$ and one decimal for the ML $(0.1 \mathrm{~N})$. $\alpha$ was then also calculated by the same author with an accuracy mathematically fixed again at one decimal $\left(0.1^{\circ}\right)$ place.

\section{Statistical evaluation}

A post-hoc power analysis was performed for opening angles, DML and ML. Non-parametric Wilcoxon test was performed to compare opening angles, DML and ML between $\mathrm{HK}+$ and HK- groups. $p<0.05$ was considered a threshold of statistical significance. To distinguish maximal angulation $>5 \pm 3^{\circ}$, opening $>10 \pm 6 \mathrm{~mm}$ and Load $>40 \pm 30$ Newtons before breakage between pairs, 5 pairs of knees were required by our sample evaluation. All calculations assumed two-sided test with alpha of 0.05 and 1-Beta of 0.8 .

\section{Results}

Analysing the graphical coordinates of the ML and the DML, the final results were obtained (Table 1). On average, opening angles were found $339 \%$ greater in the $\mathrm{HK}+$ compared to the HK- $(P=0.019)$. Indeed, $\alpha$ was on average at $9.9^{\circ}$ in the $\mathrm{HK}+$ compared to $2.9^{\circ}$ in the $\mathrm{HK}$ - group (Table 2). In the same manner, tibias of the HK+ showed a $265 \%$ increase for the DML compared to tibias of the HK- group $(P=0.05)$. Finally, a significant increase of $882 \%(P=0.04)$ was found for the ML again between the two groups. All these results are detailed in Table 2.

For each tibia, the cutting guide was accurately placed according to the pre-OWHTO planning provided and the osteotomy was performed as planned. During the second part of the osteotomy cut (PsCG removed, HK still in place), the sawblade reached the HK and could not cut further. After Mechanical testing, an evaluation of the distal part of the osteotomy made by 2 of the authors confirmed that the HK
Table 1 Summary of the results obtained and calculated for each pair of knee tested; d: distance between cut (saw-print) and hinge lateral cortex

\begin{tabular}{|c|c|c|c|c|c|c|c|c|c|c|}
\hline \multirow[t]{2}{*}{ Mechanical parameters } & \multicolumn{2}{|c|}{ Specimen 1} & \multicolumn{2}{|c|}{ Specimen 2} & \multicolumn{2}{|c|}{ Specimen 3} & \multicolumn{2}{|c|}{ Specimen 4} & \multicolumn{2}{|c|}{ Specimen 5} \\
\hline & HK- & $\mathrm{HK}+$ & HK- & $\mathrm{HK}+$ & HK- & $\mathrm{HK}+$ & HK- & $\mathrm{HK}+$ & HK- & $\mathrm{HK}+$ \\
\hline$d(\mathrm{~mm})$ & 9.2 & 9.4 & 10.1 & 10.3 & 10.9 & 11.2 & 10.3 & 10 & 11.4 & 11.6 \\
\hline $\mathrm{ML}(\mathrm{N})$ & 10.1 & 8.4 & 1.0 & 47 & 2.9 & 117.7 & 6.4 & 12.6 & 7.3 & 14.1 \\
\hline DML (mm) & 12.2 & 31.4 & 3.1 & 16.7 & 7.5 & 31.2 & 6.1 & 6.8 & 8.6 & 6.3 \\
\hline$\alpha\left(^{\circ}\right)$ & 4.4 & 15.7 & 1.1 & 6.0 & 2.7 & 12.6 & 2.7 & 3.0 & 3.8 & 2.7 \\
\hline
\end{tabular}

$M L$ maximum load before breakage of the hinge, $D M L$ displacement corresponding at the ML before breakage of the hinge, $\alpha$ angulation permitted before breakage of the hinge, $H K$ - no hinge K-wire group, $H K+$ Hinge K-wire group 
Table 2 Summary of maximum loads, DCM and mean allowable angulations for all knees with and without pins

\begin{tabular}{lllllll}
\hline $\begin{array}{l}\text { Mechanical param- } \\
\text { eters at failure }\end{array}$ & Mean (SD) & & Mean difference & 95\% IC of difference & Absolute difference & $P$ value \\
\cline { 2 - 4 } & HK+ & HK- & & & \\
\hline ML (N) & $48.3(40)$ & $5.5(4.2)$ & 42.9 & {$[6.6-97.1]$} & $+882 \%$ & 0.04 \\
DML (mm) & $19.8(11.4)$ & $7.5(3.3)$ & 12.3 & {$[7.2-17.2]$} & $+265 \%$ & 0.05 \\
$\alpha\left({ }^{\circ}\right)$ & $9.9(5.2)$ & $2.9(1.3)$ & 7 & {$[3.4-9.5]$} & $+339 \%$ & 0.02 \\
\hline
\end{tabular}

$M L$ maximum load before breakage of the hinge, $D M L$ displacement corresponding at the ML before breakage of the hinge, $\alpha$ angulation permitted before breakage of the hinge, $H K$ - no hinge K-wire group, $H K+$ Hinge K-wire group, $S D$ standard deviation

stopped our sawblade in every tibia and the HK merged at $10 \pm 1 \mathrm{~mm}$ from the lateral cortex of the tibia. The intra and extra-observer correlation was found to be $0.96 / 0.89$ for intra/inter-observer Spearman test.

\section{Discussion}

The main finding of this study demonstrated that positioning a K-wire intersecting the cutting plane at the theoretical lateral hinge location $1 /$ limits the cut depth, thus preserving sufficient lateral hinge thickness. 2 /increased the mechanical resistance of the hinge to failure. In this in-vitro study, influence of the $\mathrm{HK}$ on preserving the lateral hinge during the OWHTO opening was assessed into matched pairs of tibias coming from 5 subjects, allowing equivalent testing conditions into each pair. As each tibia underwent the same preparation process, and the location of the cutting plane and HK were defined based-on Patient-specific cutting guides, the study design only compared the influence of the HK presence during OWHTO opening. Subjects of the $\mathrm{HK}+$ group tolerated much higher ML and DML than those of the $\mathrm{HK}$ - group. In the same way, the angulation $\alpha$ allowed at the maximum load before breakage was greater when using a hinge K-wire during OWHTO opening.

Recently, Lee et al. [15] proved that the incidence of lateral hinge fractures of the knee after OWHTO was significantly higher on CT scans than on plain radiographs. The medial opening gap width was the only predictor of lateral hinge fractures, suggesting a need for care when spreading the gap to avoid lateral hinge fractures. On the other hand, Lee et al. [13] showed that surgical experience may improve the surgeon's competency in prevention of lateral hinge fractures, highlighting the need to be trained and experienced to fully respect the lateral tibial hinge. Moreover, the pivotal role of a competent lateral tibial hinge in tibial osteotomies in terms of bone consolidation and maintenance of angular correction in all 3 planes has been widely reported $[5,18$, 24, 27]. These studies suggest that the lateral tibial hinge must be kept unscathed. However, to our knowledge and to date, very few studies $[6,9]$ have investigated the resistance of this tibial hinge to fracture and/or on potential operative strategies to improve this mechanical resistance. In their numerical study, Diffo Kaze et al. [6] focused their study on the effect of hinge drilling to improve the resistance to fracture during OWHTO. Their results confirm that, for tibial varus correction osteotomies $<5^{\circ}$, drilling a hole at the end of the osteotomy reduces stresses in the lateral hinge and increases the critical angle before failure of the hinge but only for corrections inferior to $5^{\circ}$. Indeed, for osteotomies superior to $9 \mathrm{~mm}$ of opening in cadaveric specimens, Bujnowski et al. [4] recently demonstrated that a pilot hole showed no significant decrease in the strains experienced at the lateral cortex, nor did it reduce the risk of fracture of the lateral tibial hinge. According to Diffo Kaze et al. [6], the ductility of the cortical bone is the decisive parameter for the critical angle of opening, defined as the angle from which the tibial hinge breaks. These results, go in the same direction as ours: in the two studies, angulation, vertical displacement and maximum load before breakage were all greater when the hinge was protected before applying opening load. The maximal angular deformation seems to be greater using the HK method described compared to hinge drilling method by Diffo Kaze et al. [6] (fractures occurred at a mean of $9.9^{\circ}$ in this study and always $<5^{\circ}$ for all Diffo Kaze [6] et al. study's specimens). Moreover, it is important to note that the Cut K-wire was situated just below the cutting plane, so when viewing the projection of both $\mathrm{K}$-wires in the frontal plane (antero-posterior flouroscopy), the location of the hinge was just above this Cut K-wire, and just lateral to the hinge K-wire. Neither of these two K-wires follow the same placement as the pilot hole described by Diffo Kaze [6] et al. and Bujnowski et al. [6]. In this manner, the lateral tibial hinge was kept unscathed during our experiment, whereas in comparison it was drilled in the one described by Diffo Kaze et al. [6]. For each tibia, the HK limited the cutting depth by leading the saw to "touch but never to breach the HK". In this manner, the K-wire acted as a constraint to the depth of the osteotomy cut allowing preservation of a lateral tibial hinge by a width of $10 \mathrm{~mm}$.

To our knowledge, no reliable method to keep the hinge unscathed yet exists. The sole use of flouroscopic images does not prevent hinge fractures and does not accurately ensure the precision required to stop the saw from damaging 
the hinge. Using a traditional surgical technique, an incidence of almost $30 \%$ of hinge fractures has been reported, most of which are not recognized during surgery but rather identified at the radio-clinical 6 week follow-up [5]. Even so, for Dexel et al. [5], locking plates with extended diaphyseal fixation decreases the importance of an intact lateral hinge. Kim et al. [11] demonstrated in their retrospective study that lateral hinge fractures, when occurring in OWHTO using a medial locking plate, showed no radiological changes or functional deterioration during midterm follow-up when compared to a group that endured OWHTO with the same plates but with no lateral hinge breakage appearing peri or post operatively. However, our study investigates a potential solution to protect the hinge intra-operatively and does not study the measures required when the hinge is fractured during or after the index surgery. In the same way, even if larger plates decrease the importance of protecting the hinge, the advantage of mini-invasive surgery remains important and should encourage the surgeon to deal and anticipate hinge failure with a minimally invasive approach. Lee et al. [14] also showed that further evaluation with CT scanning was almost obligatory after OWHTO because of its higher detection rate $(24.5 \%)$ of lateral tibial hinge compared with simple radiographs (16\%). They importantly recognized the coronal osteotomy slope was steeper and the opening gap was larger when the hinge was broken. They concluded their work by stating that lateral tibial hinge fractures should be managed conservatively to prevent postoperative correction loss. CAS-OWHTO [2] does not prevent hinge failure, but rather permits to optimize the position of the hinge's Axis, thus avoiding hinge related complications (Hinge Fractures, Bone healing impairment and secondary loss of correction).

This experiment has some limitations. First, the cohort used is small with 5 samples in each group. However, our study was powered to distinguish clinically relevant differences between the two groups [23]. Moreover, the study model is based on tibial pairs, which allows direct comparison and limits potential confounding factors such as cadaveric bone quality, specimens age, and conservation process, as the only difference between the groups was the positioning of a hinge K-wire during osteotomy opening. As an ex-vivo study, it only approximates in-vivo conditions. The authors appreciate that this is mechanical testing on bone rather than bone and the surrounding soft tissues which may improve the stability of the lateral hinge [12].

This comparative cadaveric study demonstrates that an improvement of the lateral hinge resistance to fracture during osteotomy opening can be obtained with an addition of a K-wire intersecting the cutting plane at the theoretical lateral hinge location. Therefore, the protective nature of the K-wire in-vivo, if applied in reality could substantially decrease the rate of hinge facture and prevent subsequent OWHTO adverse outcomes.

\section{Conclusion}

This comparative cadaveric study showed that an improvement of the lateral hinge resistance to fracture during osteotomy opening could be obtained by the addition of a K-wire intersecting the cutting plane at the theoretical lateral hinge location.

Funding No funding was received to complete this study protocol.

\section{Compliance with ethical standards}

Conflict of interest Mathias Donnez is a paid employee of Newclip technologies Matthieu Ollivier, Sebastien Parratte and Mathieu Ehlinger are paid consultant for new-clip technologies Jean-noel Argenson and sebastien Parratte are paid consultant and receive royalties for Zimmer Biomet Matthieu Ollivier is paid consultant for Stryker and Arthrex. Akash Sharma, Patrick Chabrand and Edouard Dessyn have no conflict of interest to disclose.

Ethical approval All procedures performed in studies involving human participants were in accordance with the ethical standards of the institutional and/or national research committee and with the 1964 Helsinki declaration and its later amendments or comparable ethical standards.

Informed consent This study involved de-identified human anatomical specimens.

\section{References}

1. Ahlbäck S, Rydberg J (1980) X-ray classification and examination technics in gonarthrosis. Lakartidningen 77:2091-2093, 2096

2. Bae DK, Ko YW, Kim SJ, Baek JH, Song SJ (2016) Computerassisted navigation decreases the change in the tibial posterior slope angle after closed-wedge high tibial osteotomy. Knee Surg Sports Traumatol Arthrosc 24:3433-3440

3. Bae DK, Park CH, Kim EJ, Song SJ (2016) Medial cortical fractures in computer-assisted closing-wedge high tibial osteotomy. Knee 23:295-299

4. Bujnowski K, Getgood A, Leitch K, Farr J, Dunning C, Burkhart TA (2018) A pilot hole does not reduce the strains or risk of fracture to the lateral cortex during and following a medial opening wedge high tibial osteotomy in cadaveric specimens. Bone Jt Res 7:166-172

5. Dexel J, Fritzsche H, Beyer F, Harman MK, Lützner J (2017) Open-wedge high tibial osteotomy: incidence of lateral cortex fractures and influence of fixation device on osteotomy healing. Knee Surg Sports Traumatol Arthrosc 25:832-837

6. Diffo Kaze A, Maas S, Hoffmann A, Pape D (2017) Mechanical strength assessment of a drilled hole in the contralateral cortex at the end of the open wedge for high tibial osteotomy. J Exp Orthop $4: 23$

7. Flecher X, Parratte S, Aubaniac J-M, Argenson J-NA (2006) A 12-28-year followup study of closing wedge high tibial osteotomy. Clin Orthop Relat Res 452:91-96

8. Han S-B, Park H-J, Lee D-H (2016) Ability of an intentionally smaller anterior than posterior gap to reduce the sagittal tibial 
slope in opening wedge high tibial osteotomy. BMC Musculoskelet Disord 17:216

9. Jang YW, Lim D, Seo H, Lee MC, Lee O-S, Lee YS (2018) Role of an anatomically contoured plate and metal block for balanced stability between the implant and lateral hinge in open-wedge high-tibial osteotomy. Arch Orthop Trauma Surg 138:911-920

10. Jo H-S, Park J-S, Byun J-H, Lee Y-B, Choi Y-L, Cho S-H, Moon D-K, Lee S-H, Hwang S-C (2018) The effects of different hinge positions on posterior tibial slope in medial open-wedge high tibial osteotomy. Knee Surg Sports Traumatol Arthrosc 26:1851-1858

11. Kim K-I, Kim GB, Kim HJ, Lee SH, Yoon W-K (2018) Extraarticular lateral hinge fracture does not affect the outcomes in medial open-wedge high tibial osteotomy using a locked plate system. Arthrosc J Arthrosc Relat Surg 34:3246-3255

12. Lee D-H, Park S-C, Park H-J, Han S-B (2016) Effect of soft tissue laxity of the knee joint on limb alignment correction in open-wedge high tibial osteotomy. Knee Surg Sports Traumatol Arthrosc 24:3704-3712

13. Lee DK, Kim KK, Ham CU, Yun ST, Kim BK, Oh KJ (2018) The learning curve for biplane medial open wedge high tibial osteotomy in 100 consecutive cases assessed using the cumulative summation method. Knee Surg Relat Res 30:303-310

14. Lee O-S, Lee YS (2018) Diagnostic value of computed tomography and risk factors for lateral hinge fracture in the open wedge high tibial osteotomy. Arthroscopy 34:1032-1043

15. Lee S-S, Celik H, Lee D-H (2018) Predictive factors for and detection of lateral hinge fractures following open wedge high tibial osteotomy: plain radiography versus computed tomography. Arthroscopy 34:3073-3079

16. Lee YS, Lee MC, Kang SG, Elazab A, Oh WS (2016) Openwedge high tibial osteotomy using a protective cutting system: technical advancement for the accuracy of the osteotomy and avoiding intraoperative complications. Arthrosc Tech 5:e7-e10

17. Lee YS, Moon GH (2015) Comparative analysis of osteotomy accuracy between the conventional and devised technique using a protective cutting system in medial open-wedge high tibial osteotomy. J Orthop Sci 20:129-136

18. Lobenhoffer P (2014) [Importance of osteotomy around to the knee for medial gonarthritis. Indications, technique and results]. Orthopade 43:425-431

19. Martin R, Birmingham TB, Willits K, Litchfield R, Lebel M-E, Giffin JR (2014) Adverse event rates and classifications in medial opening wedge high tibial osteotomy. Am J Sports Med 42:1118-1126

20. Munier M, Donnez M, Ollivier M, Flecher X, Chabrand P, Argenson J-N, Parratte S (2017) Can three-dimensional patient-specific cutting guides be used to achieve optimal correction for high tibial osteotomy? Pilot study. Orthop Traumatol Surg Res 103:245-250

21. Nakamura R, Komatsu N, Fujita K, Kuroda K, Takahashi M, Omi R, Katsuki Y, Tsuchiya H (2017) Appropriate hinge position for prevention of unstable lateral hinge fracture in open wedge high tibial osteotomy. Bone Jt J 99-B:1313-1318

22. Nawas HT, Vansadia DV, Heltsley JR, Suri M, Montgomery S, Jones DG (2016) Factors affecting the union of opening wedge high tibial osteotomy using a titanium wedge plate. Ochsner J 16:464-470

23. Ogden S, Mukherjee DP, Keating ME, Ogden AL, Albright JA, McCall RE (2009) Changes in load distribution in the knee after opening-wedge or closing-wedge high tibial osteotomy. J Arthroplasty 24:101-109

24. Saragaglia D, Chedal-Bornu B, Rouchy RC, Rubens-Duval B, Mader R, Pailhé R (2016) Role of computer-assisted surgery in osteotomies around the knee. Knee Surg Sports Traumatol Arthrosc 24:3387-3395

25. Stoffel K, Stachowiak G, Kuster M (2004) Open wedge high tibial osteotomy: biomechanical investigation of the modified Arthrex Osteotomy Plate (Puddu Plate) and the TomoFix Plate. Clin Biomech Bristol Avon 19:944-950

26. Takeuchi R, Ishikawa H, Kumagai K, Yamaguchi Y, Chiba N, Akamatsu Y, Saito T (2012) Fractures around the lateral cortical hinge after a medial opening-wedge high tibial osteotomy: a new classification of lateral hinge fracture. Arthroscopy 28:85-94

27. Weng P-W, Chen C-H, Luo C-A, Sun J-S, Tsuang Y-H, Cheng C-K, Lin S-C (2017) The effects of tibia profile, distraction angle, and knee load on wedge instability and hinge fracture: a finite element study. Med Eng Phys 42:48-54 